\title{
Tamsulosin bei größeren distalen Uretersteinen
}

\author{
Für den spontanen Abgang größerer distaler Uretersteine $>5 \mathrm{~mm}$ kann der Einsatz von \\ Tamsulosin erwogen werden. Bei kleineren Steinen hat der Alpha-Blocker keinen Effekt.
}

\begin{abstract}
_ Nierensteinkoliken betreffen über die gesamte Lebenszeit etwa $12 \%$ der Männer und 6\% der Frauen. Steine $>5 \mathrm{~mm}$ Durchmesser erfordern häufig eine Intervention. Zur medikamentösen Therapie werden Alpha-Blocker empfohlen, insbesondere Tamsulosin. Die Studienlage dazu ist jedoch dürftig.

In einer randomisierten, doppelblind geführten und placebokontrollierten Multicenterstudie wurden erwachsene Patienten mit computertomografisch nachgewiesenen Uretersteinen über 28 Tage hinweg entweder mit 0,4 mg Tamsulosin oder Placebo behandelt. Primärer Studienendpunkt war der Anteil abgegangener Steine nach vier Wochen und die Zeit bis zum Steinabgang.
\end{abstract}

403 Patienten konnten eingeschlossen werden. $81,4 \%$ waren Männer, das mediane Alter lag bei 46 Jahren. In der Verumgruppe war die mediane Steingröße 4,0 mm, in der Placebogruppe 3,7 mm. Bei 316 Patienten konnte nach 28 Tagen ein CT angefertigt werden.

Unter Tamsulosin war bei $87,0 \%$ der Patienten das Konkrement abgegangen, in der Placebogruppe bei $81,9 \%$. Dieser Unterschied war statistisch nicht signifikant. In der Untergruppe von Patienten mit 5-10 mm Steingröße gelang unter Tamsulosin ein Steinabgang bei 30 von 36 Patienten $(83,3 \%)$, in der PlaceboGruppe nur bei 20 von 41 Patienten (61\%). Daraus ergibt sich eine Number Needed to Treat von 4,5.
Bei den urologischen Interventionen, der Zeit bis zum Steinabgang, beim Schmerzscore oder beim Bedarf an Analgetika waren keine signifikanten Unterschiede festzustellen.

- Furyk JS, Chu K, Banks Cet al. Distal ureteric stones and tamsulosin: a double-blind, placebo-controlled, randomized, multicenter trial. Ann Emerg Med. 2016;67:86-95

\section{KOMMENTAR}

Mit der gut gemachten Studie an einer groBen Anzahl von Patienten dürfte die Frage der Förderung des Uretersteinabgangs durch Tamsulosin endgültig beantwortet sein. Bei Steinen unter $5 \mathrm{~mm}$ bringt die Maßnahme keine Vorteile. Bei größeren Steinen kann man sie durchaus in Erwägung ziehen.

Prof. Dr. med. H. S. Füeß।

\section{Pneumoperitoneum verursacht erhebliche Schmerzen}

Eine 25-jährige Frau litt seit drei Tagen an Bauchschmerzen und Erbrechen. Sie war exsikkiert und hatte Fieber. An der rechten Flanke bestand ein erheblicher Druck- und Loslassschmerz. Sie hatte eine Tachykardie und Tachypnoe, Blutdruck und $\mathrm{O}_{2}$-Sättigung waren aber normal. Die Routine-Laborparameter waren massiv verändert: BKS $122 \mathrm{~mm} / \mathrm{h}$, Leukozyten

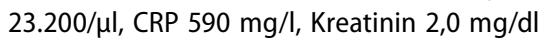
und Glukose $576 \mathrm{mg} / \mathrm{dl}$. In der Blutgasanalyse bestand eine metabolische Azidose, im Urin fanden sich Ketonkörper und eine Glukosekonzentration von $500 \mathrm{mg} / \mathrm{dl}$. $\beta$-hCG war negativ. Man vermutete eine neu aufgetretene diabetische Ketoazidose, wahrscheinlich aufgrund einer Sepsis.

Im Röntgen-Thorax fand sich subdiaphragmal rechts freie Luft, in der Abdomen-Übersicht eine gefleckte, umschriebene und glatt begrenzte Gasansammlung (Abb. A), die in der CT des Abdomens einer stark vergrößerten rechten Niere zugeordnet werden konnte (Abb. B). Unter der Diagnose einer emphysematösen Pyelonephritis wurde die Patientin über zwei Wochen stationär mit Meropenem i.v. behan-
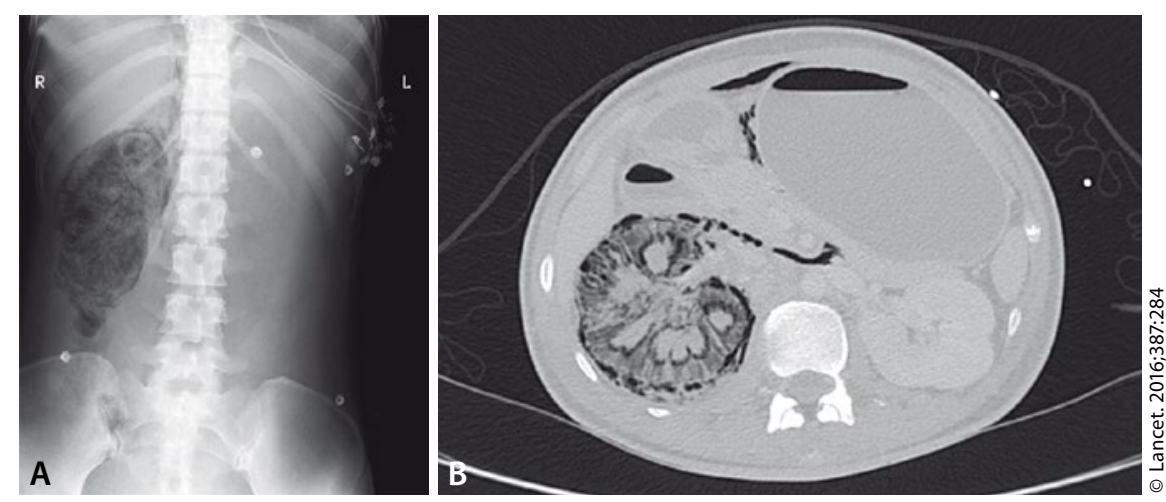

Gefleckte, umschriebene und glatt begrenzte Gasansammlung im rechten oberen Quadranten im Röntgen-Abdomen (A), vergrößerte rechte Niere im CT (B).

delt. Daran anschließend erhielt sie ambulant Ertapenem i.v. In der fünften Krankheitswoche entstand ein perinephritisches Empyem, das drainiert werden musste. Im Eiter wuchsen Shigatoxin-produzierende E. coli, die auf Ertapenem empfindlich waren. Nach fünf Monaten fortlaufender antibiotischer Therapie musste die rechte Niere doch entfernt werden, da sich immer wieder Eiter bildete.
Die emphysematöse Pyelonephritis führt häufig zur vollständigen Zerstörung der Niere. Die Mortalität liegt zwischen 18 und 69\%, wobei eine frühzeitige Behandlung essenziell ist. Das Gas war bei der Patientin wohl durch das Peritoneum hindurch in den intraperitonealen Raum diffundiert.

- Prof. Dr. med. H. S. FüeßI

- Hateley C et al. (jimbuckley@nhs.net) Pneumoperitoneum, a urological source. Lancet. 2016;387:284 